\title{
AlphaFold Models Illuminate Half of Dark Human Proteins
}

\author{
Jessica L. Binder ${ }^{1}$, Joel Berendzen ${ }^{2}$, Amy O. Stevens ${ }^{3}$, Yi He ${ }^{1,3}$, Jian Wang ${ }^{4}$, Nikolay V. \\ Dokholyan $^{4,5}$, \& Tudor I. Oprea ${ }^{1,6,7,8, *}$
}

1. Translational Informatics Division, Department of Internal Medicine, University of New Mexico, Albuquerque, NM 87131, USA.

2. GenerisBio, LLC, Santa Fe, NM 87507, USA.

3. Department of Chemistry and Chemical Biology, University of New Mexico, Albuquerque, NM 87131, USA.

4. Department of Pharmacology, Department of Biochemistry and Molecular Biology, Penn State University College of Medicine, Hershey, PA 17033, USA.

5. Department of Chemistry and Department of Biomedical Engineering, Pennsylvania State University, University Park, Pennsylvania 16802, United States.

6. UNM Comprehensive Cancer Center, Albuquerque, NM, USA.

7. Department of Rheumatology and Inflammation Research, Institute of Medicine, Sahlgrenska Academy at the University of Gothenburg, Gothenburg, Sweden.

8. Novo Nordisk Foundation Center for Protein Research, Faculty of Health and Medical Sciences, University of Copenhagen, Copenhagen, Denmark.

*Corresponding author: Oprea, T.I. | email toprea@ salud.unm.edu | Tel +15059254756

\section{ABSTRACT:}

We investigate the use of confidence scores to predict the accuracy of a given AlphaFold model for drug discovery. Predicted accuracy is improved by eliminating confidence scores below 80, due to effects of disorder. $95 \%$ of models corresponding to a set of recent crystal structures are accurate at the fold level. Conformational discordance in the training set has a more significant effect on accuracy than sequence divergence. We propose criteria for models and residues that are possibly useful for virtual screening, by which AlphaFold provides models for half of understudied (dark) human proteins and two-thirds of residues in those models. 


\section{INTRODUCTION}

About half of Americans answering a 2020 survey would not get in an AI-driven taxi, and about threequarters of them believed AI (artificial intelligence) cars were "not ready for primetime" [1]. Whether driving a vehicle or discovering new medicines, trust in AI depends on accumulated community experience and the consequences of errors in specific cases. There are over 20,000 protein-coding genes in the human genome [2-4]. Of these, 7074 have experimentally determined structures deposited in the Protein Data Bank (PDB) as of July 2021 [5]. Only 670 human proteins are therapeutically targeted by medicines, comprising the "drugged genome" [6]. Significant areas of biology remain potentially amenable to drug discovery [7]. Initiatives like "Illuminating the Druggable Genome" [8], OpenTargets [9], and Target 2035 [10] are exploring novel therapeutic opportunities in the "druggable" genome.

DeepMind described [11,12] AF2 (AlphaFold version 2.0), an AI method that predicts overall 3D structures of proteins. More than 350,000 AF2 structural models (including models of nearly every human protein) are now publicly accessible [12]. DeepMind garnered worldwide attention with their decisive win of the Critical Assessment of Techniques for Protein Structure Prediction, CASP14 [13]. Currently, scientists are assessing the impact of AF2 on research, including how much AF2 models expand the druggable genome.

Winning CASP14 presents a set of challenges specific to protein folding. However, protein 3D models do not often play a crucial role in drug discovery. The notion of trust in AF2 models is illustrated with a histogram of atomic Root-Mean-Square Deviations ( $a R M S D)$ on $\mathrm{C}_{\text {曰 }}$ atoms for crystal structures deposited in the PDB since AF2 was trained (Fig. $2 a$ in [11]). It shows that AF2 produces high-quality folds in two-thirds of cases. However, the overall accuracy of a given AF2 model was not discussed. Local confidence scores (predicted Local Distance Difference Test, $p L D D T$ ) show a 95\% perresidue correlation with experimentally-derived $L D D T$ values [14] over the same proteins. AF2 model confidence evaluation is needed in the drug discovery context, given the non-local nature of $a R M S D$, the inherent selection bias of recent PDB structures, and the lack of any overall confidence-in-accuracy measure that can be calculated for individual models. Here, we discuss the issue of trust in AF2 models by addressing disorder, divergence, discordance, and druggability. 


\section{Disorder dominates confidence scores below 80}

More than $30 \%$ of eukaryotic proteins contain one or more intrinsically disordered regions, IDRs [1521]. Disorder is reflected in confidence scores as regions with low $p L D D T$ [12]. Figure 1 displays the distributions of the $p L D D T$ scores reported by AF2 for resolved/ordered and unresolved/disordered regions of crystal structures deposited in the PDB since AF2 was trained (the "post-AF2 test set", see Supplemental Information). On this set of structures, ordered regions most frequently show $p L D D T$ scores greater than 80, while IDRs have a broad distribution of pLDDT scores, with about $40 \%$ of unresolved regions falling below a $p L D D T$ score of 50. From this analysis, we conclude that pLDDT confidence scores below 80 are more indicative of disorder than of confidence in the accuracy of ordered structures.

\section{Divergence has a minor effect on model accuracy}

A problem with the 6-bin histogram used to estimate the distribution of model accuracies [11] is that $a R M S D$ is a non-local measure. If a model is incorrect at the fold level, the expectation value of $a R M S D$ scales with the radius of gyration. Thus, a model with $30 \AA$ $a R M S D$ against the experimental structure could be consistent with an entirely misfolded domain of around 1000 residues in length [22] or simply with rotation of a smaller domain about a single residue. To characterize different effects on model accuracy, we down-selected the post-AF2 test set to 1,779 models that can be aligned with a corresponding experimental structure (see Supplemental Information) and used them to evaluate allatom and backbone measures. pLDDT correlates poorly with $\log (a R M S D)$ on this set: Spearman rank correlation coefficient is 0.43 on the median (Supplemental Figure S1A). Truncating the range of pLDDT over which the median is calculated with a floor of 80 slightly improves the coefficient to -0.48 (Supplemental Figure S1B). We refer to the per-model median value of $p L D D T$ scores greater than 80 as $p L D D T_{80}$.

Next, we split this down-selected test set into two pairs of subsets. The first pair explored high $\left(p L D D T_{80}>90\right)$ or low $\left(p L D D T_{80}<88\right)$ confidence scores. The second pair explored high (in clusters at $100 \%$ identity for over $80 \%$ of the length) or low (out of clusters at $5 \%$ identity for over $80 \%$ of the length) sequence identity to structures previously in the PDB. Cutoff values in these pairings were chosen to give maximal differences while maintaining roughly comparable fractions of the test set in the 
two arms of each pairing. We calculated distributions on $\log (a R M S D)$ and on the all-atom LDDT [14] for each of the subsets (Figure 2).

The aRMSD metric is not well-suited for characterizing structural models because its non-local nature tends to exaggerate the effects in small number backbone angle changes [23]. The lack of a highdifference in the low-identity $L D D T$ distribution, together with the suppression of the high-difference tail in the low-confidence distribution, suggest that most differences between model and structure are in a few local coordinates, rather than many. Using LDDT as the accuracy measure improved Spearman's correlation on $p L D D T_{80}$ to 0.60 (Supplemental Figure S2).

Less than $1 \%$ of the high-confidence distribution appears in the range consistent with fold-level inaccuracies at $L D D T<50$. Distributions for the low-similarity, high-confidence, and high-identity subsets are the same, with the caveat afforded by the paucity of low-similarity models. But the $4 \%$ of models in the low-confidence distribution are distinctly worse than the other subsets. These observations suggest that AF2 produces models that are correct at the fold level more than $95 \%$ of the time, better than the previous two-thirds estimate [11].

\section{Discordance may limit model accuracy}

The similar distribution between the high-confidence and the high-identity subsets (Figure 1) suggests that the main driver of the accuracy of most models is conformational change among multiple structures of nearly-identical sequences already in the PDB. Multiple PDB entries can reflect different conformations. Which protein conformation is more druggable depends on the clinical need associated with a particular disease state. AF2's training algorithm handles multiple structure files in the PDB with the same primary sequence by down-selecting among them [11]. Structural details relevant for drug discovery, such as prosthetic groups, ion binding, and protons are not included, and AF2 models also exclude post-translational modifications.

An extreme conformational discordance case is calmodulin (Figure 3), a kinase that upon binding $\mathrm{Ca}^{++}$ions changes from a globular to a dumb-bell shape by virtue of two hinge residues [24]. In the calmodulin AF2 model, the effects of discordance due to both conformations being present in the training set are reflected by a region of low-confidence scores at the hinge residues. Different AF2 runs yield slightly different results, but the resulting models split the difference between the two states (e.g., the AF2 model represented in grey on the top right side of Figure 3). This suggests that discordance 
among structures in the AF2 training set can result in composite models that may not necessarily accurately reflect the basic structure of any of the structures in the PDB, even for identical sequences.

\section{Druggability: Are AF2 models ready for virtual screening?}

Known protein conformational changes (as shown in Figure 3) can help us assess the effects of model accuracy on AF2 model readiness for target-based virtual screening (TBVS). If the model needs to be as close to the crystal structure as deoxy-myoglobin is to carboxy-myoglobin [25], only a tiny fraction of the AF2 models would be suitable for TBVS. If the model needs to be as close as R-state is to T-state hemoglobin, AF2 models may be suitable for characterizing allosteric sites [26]. A more typical TBVS example, where accuracy needs to be similar in capturing conformational changes, is when ERK2 is doubly phosphorylated. Given this example, a practical lower bound of global $p L D D T$ of 80 could serve as basis for a model to likely be TBVS-ready. A value of $p L D D T$ of 80 indicates a $68 \%$ likelihood of sidechain rotamers falling into the correct hemisphere (Fig 2B in [11]). Surfaces formed by two adjacent residues with $p L D D T \geq 80$ are very close to the $50 \%$ accuracy limit if rotamer errors are independent. Having previously introduced $p L D D T_{80}$, we set $p L D D T_{80} \geq 91.2$ as criterion for assessing AF2 model quality, combined with the fraction of protein length for which this holds true ( $p L D D T_{80}$ frac) to evaluate TBVS potential; see Supplemental Information. These criteria allow us to calculate a confusion matrix (see Supplemental Figure S2) that gives the sensitivity (true positive rate of classification) of $90.1 \%$ and a precision (positive predictive value of classification) of $86.3 \%$.

Given these criteria, we evaluated which AF2 models of the human understudied proteins, Tdark [7], which currently lack an experimental PDB structure might be TBVS-ready (Figure 4A). Of the set of 5592 "dark" proteins with AF2 models, 3051 (54.6\%) meet our criteria for possibly being accurate enough for TBVS studies (Figure 4B). Taking into account the estimated false-positive rate ( 6\% of total), this implies that AF2 provides TBVS-ready models for about half of the understudied human proteins.

\section{CONCLUSIONS}

In our opinion, future work would do well to move away from the familiar aRMSD metric of overall model-structure agreement in favor of LDDT or other local measures. The aRMSD metric suggests that 
AF2 models are worse compared to pLDDT. Structural bioinformatics would also benefit from developing measures that disambiguate the effects of disorder, discord, and divergence.

AF2 forces us to reconsider the implications of disorder on druggability because it performs well at predicting IDRs [27]. Having trust that a region is disordered versus trusting the ordered region's accuracy leads to different conclusions. Proteins containing IDRs play critical roles in many biological functions [28-35] and are associated with various diseases [36-39]. Thus, IDRs are potential targets in drug discovery [40-42]. "Disordered" does not mean "undruggable" because unique strategies for drug discovery in targets containing disordered regions are available [43]. Regions of pLDDT < 50 in an AF2 model indicate those strategies could be employed. Moreover, the existence of a structural model is neither necessary nor a sufficient condition for drug discovery. Even the use of high-quality experimental structures of the correct conformational state does not guarantee successful TBVS hits.

About 5\% of the human "dark" proteome has structures in the PDB (Supplemental Information). Cost-to-benefit analyses of whether to deploy TBVS on AF2 models remain project-dependent. However, AF2 model quality may be "good enough" for rapid deployment for over 3000 understudied human proteins. AF2 models may help de-risk protein targets through protein expression and solubility and may provide protein engineering suggestions. By identifying likely boundaries of compact domains, disordered regions, or linkers, AF2 and other methods can enable synthesis-by-domain strategies that can break large proteins into more tractable modules to be expressed or synthesized then reconstituted in-vitro. Regardless of its impact on in silico technologies, AF2 does not preclude structural biology and structure-based drug design. However, AF2 is poised to become a powerful tool in the evolving drug discovery arsenal.

Computational models are very different from experimental structures in that they can be updated on-demand with the latest improvements. Public notebooks such as ColabFold [44] facilitate the removal of disordered termini, improving sequence alignment, adding a binding partner, and calculating new models within minutes. Although not designed with protein oligomers or assemblies in mind, multiple groups are working on use of AF2 to illuminate protein-protein interactions. In 2014, it was estimated that $40 \%$ of protein structures were experimentally determined [45]. With AF2 and future improvements, structural biology, and drug discovery are about to exponentially increase with new computational tools that combine sequence evolution, structures, and ligand binding knowledge [46]. 


\section{Acknowledgements}

We acknowledge support by the National Institutes for Health IDG KMC application from the University of New Mexico (Grant No. U24CA224370), the Passan Foundation (Grant No. R35

GM134864 to N.V.D.), the National Science Foundation (Grant No. 2137558 to Y.H.), and the National Science Foundation Graduate Research Fellowship (Grant No. DGE-1939267).

\section{Resources}

We have implemented a $p L D D T_{80}$ classifier along with other useful features as a python package called rafm, which is installable via the usual mechanism of "pip install rafm" at the command line on systems with python 3.8 or greater installed. https://github.com/unmtransinfo/rafm

Supplementary Information contains pLDDT and derivative score information for the PDB subset, and for the Tdark subset of the human proteome, as well as AF2 model evaluation criteria.

\section{Conflict of Interest}

T.I.O. has received honoraria from or consulted for Abbott, AstraZeneca, Chiron, Genentech, Infinity Pharmaceuticals, Merz Pharmaceuticals, Merck Darmstadt, Mitsubishi Tanabe, Novartis, Ono Pharmaceuticals, Pfizer, Roche, Sanofi and Wyeth, and is on the Scientific Advisory Board of ChemDiv and InSilico Medicine. 


\section{FIGURES}

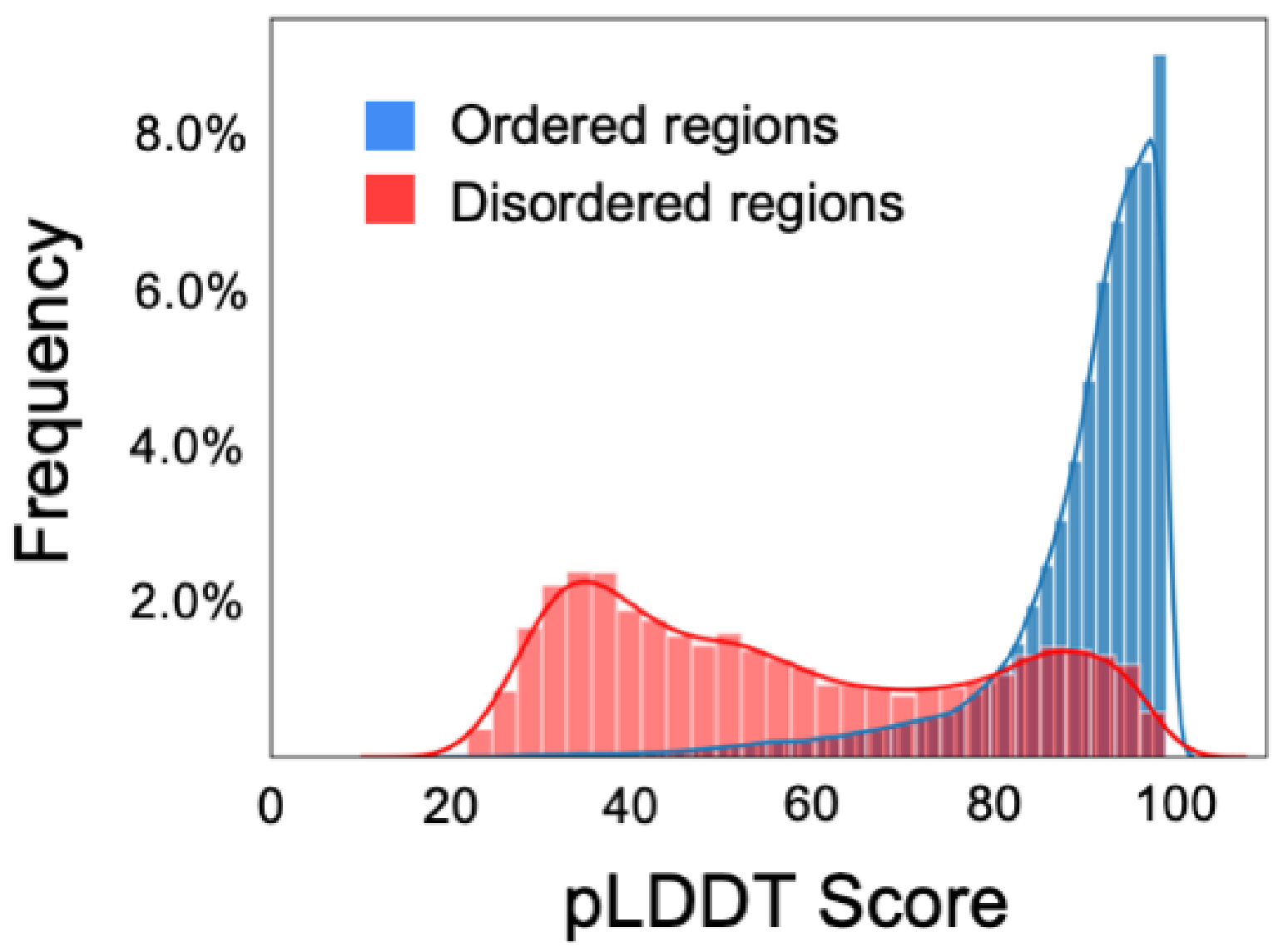

Figure 1. Distribution of AlphaFold confidence scores across ordered (blue) and disordered (red) regions. Ordered and disordered regions correspond to resolved and unresolved parts, respectively, for the post-AF2 test set. Terminal regions were not included. Ordered regions most frequently show pLDDT scores $>80 \%$. Disordered regions show a broad distribution of pLDDT scores with comparable frequencies from pLDDT scores between $20 \%$ and $90 \%$. 

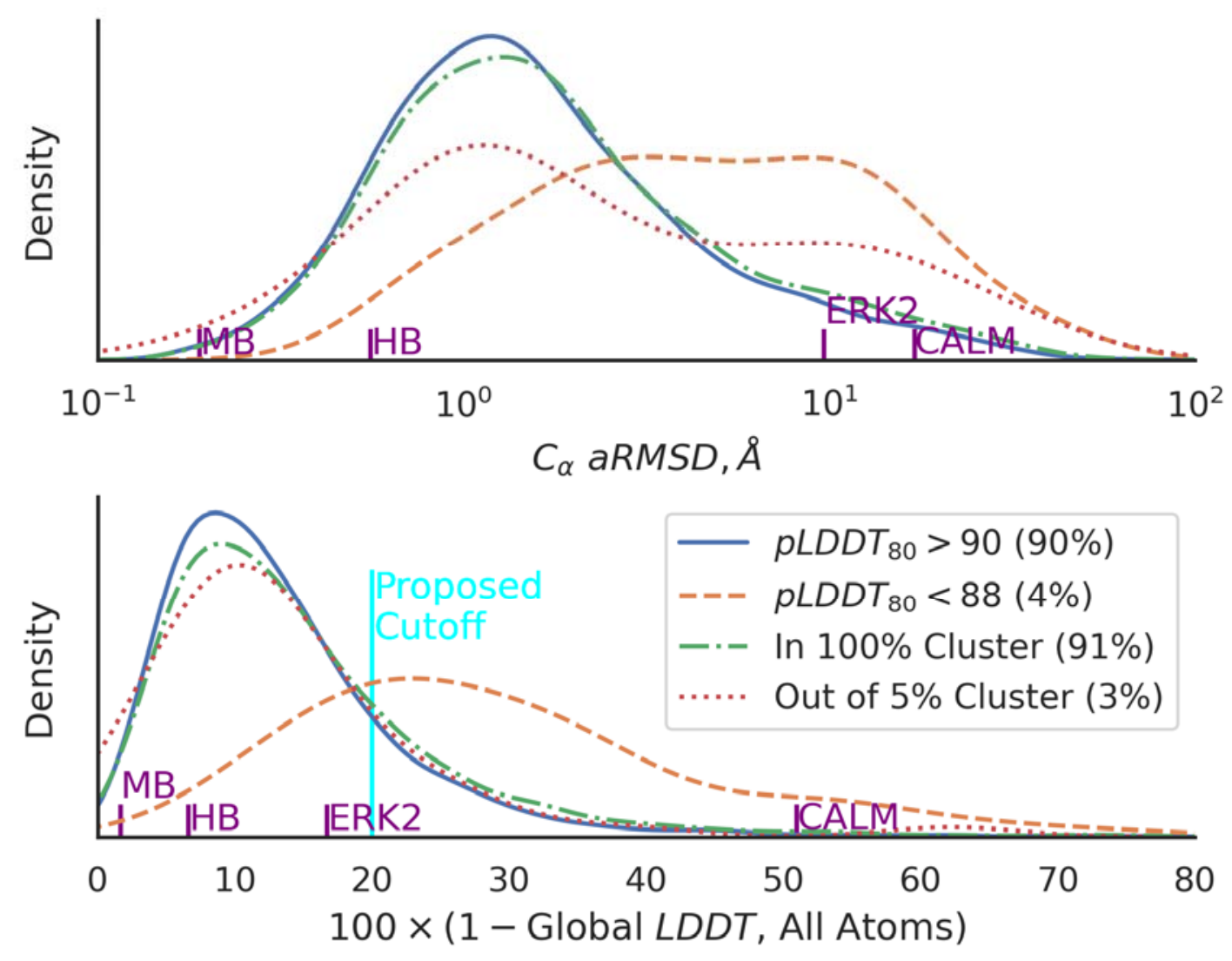

Figure 2. Kernel-density estimates of the distribution of differences between AF2 models and crystal structures using atomic Root-Mean-Square Displacements on $\mathbf{C}_{\boldsymbol{\alpha}}$ atoms (top) and Global Local Distance Difference Test metrics (bottom). These distributions were calculated from crystallographic structures that were deposited in the Protein Data Bank after the AF2 training set cut-off date of April 30, 2018. Only residues that unambiguously intersect between AF2 models deposited in EMBL [47] and crystal structures were considered, with a minimum per-chain length cutoff of 20 residues, resulting in 1810 structural models to be compared. Distributions for mean confidence levels ( $\left.p L D D T_{80}\right)$ over the raw models at or above 90 (solid blue line) and below 88 (dotted orange line) are plotted. We also clustered the PDB using mmseqs [48] to select for sequences nearly identical to an existing structure (in clusters with $100 \%$ minimum sequence identity over $80 \%$ of the longest sequence and cluster mode 2 , dot-dash green line) or decisively non-matching regions (out of 5\% minimum sequence identity, dotted red line). The high-confidence ( $\left.p L D D T_{80}>90\right)$ distribution on $\log (a R M S D)$ peaks at $1.7 \square$ aRMSD, with a long tail extending beyond $10 \AA$ at the $10 \%$ level. The low-confidence distribution on log(aRMSD) has a broad flat shape suggesting peaks at 3 and $20 \AA$. The highidentity distribution looks similar to the high-confidence distribution, while the low-identity distribution has peaks near 2 and $20 \AA$, respectively. Plotted against all-atom LDDT, the highconfidence, high-identity, and low-identity distributions look similar to each other; only the lowconfidence distribution is distinct, with a single peak at LDDT 75. Notations on the $x$-axis indicate differences between structures of ligand-free vs. ligand bound myoglobin (MB, PDB 
entries $1 A 6 N$ and $1 A 6 G) ; R$-vs. T-state hemoglobin (HB, PDB entries 6BWP and 6BWU); unphosphorylated vs. doubly-phosphorylated conformations of an extracellular signal-regulated kinase (ERK2, PDB entries 1ERK and 2ERKO); and calcium-free vs. calcium-bound calmodulin (CALM, PDB entries 1CLL and 1QX5). The cyan line shows the proposed LDDT cutoff for a structure that is likely to be useful for virtual screening.

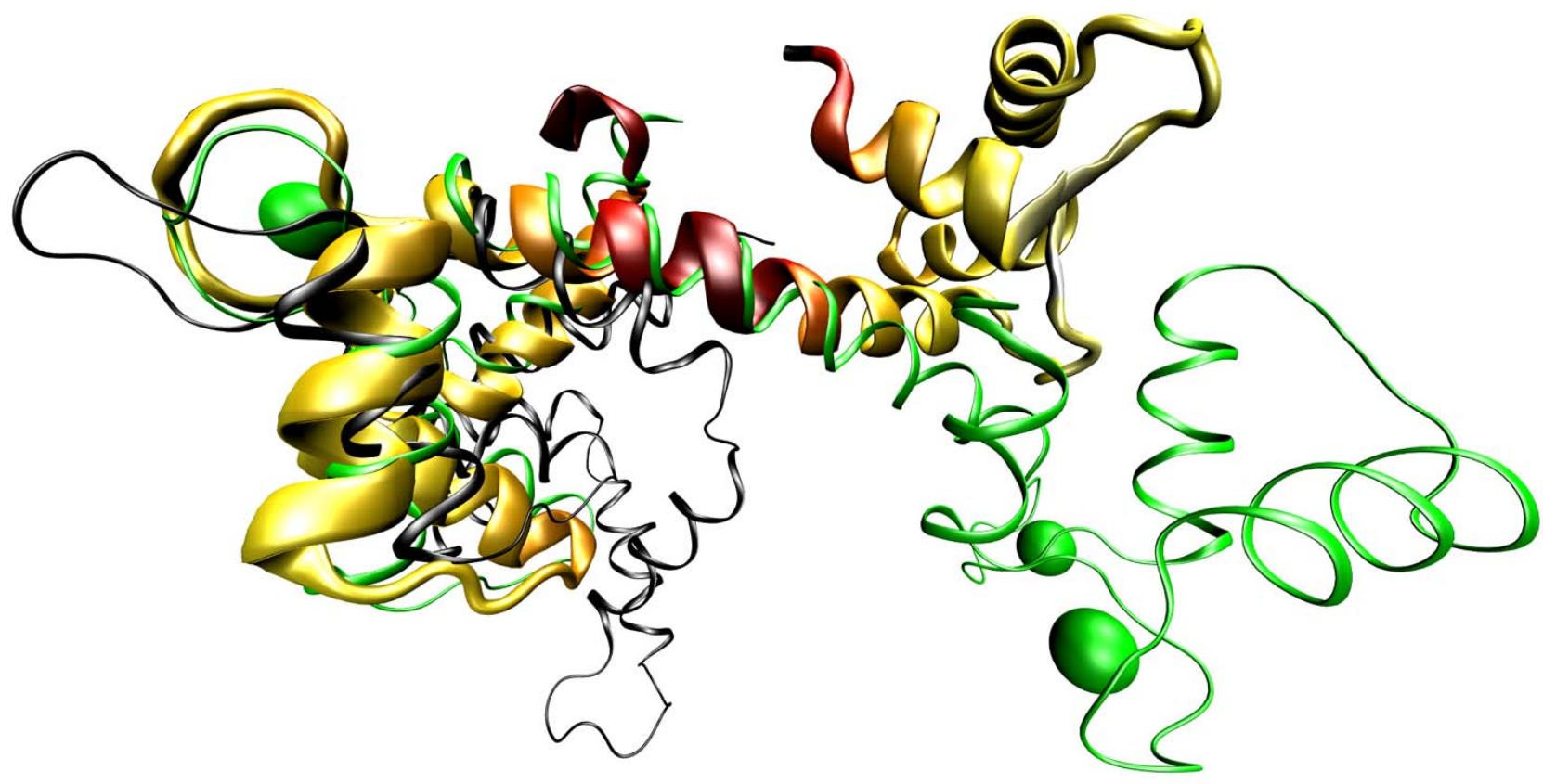

Figure 3. Comparison of discordant crystal structures of calmodulin with an AF model. The calcium-bound crystal structure (PDB entry $1 C L L$, thin green cartoon with $C a^{++}$ions as spheres), with alignments against the first half of the calcium-free crystal structure (PDB entry 1QX5, thin black cartoon) and the AlphaFold2 model (P0DP23-F1-model_v1, thick yellow-red cartoon), aligned on their $N$-terminal halves. Yellow regions of the model represent very high confidence (pLDDT > 90) residues, while dark-red regions represent very low confidence (pLDDT<50) residues. The low confidence region at the center of the AF model corresponds to a hinge where the calcium-bound and calcium-free models diverge. When aligned in this manner, aRMSD values of $7.2 \AA$ against the calcium-bound structure and $6.7 \AA$ against the calcium-free structure were obtained. When aligned across all residues, the AF model yields aRMSDs of $10 \AA$ against the calcium-bound structure and $17 \AA$ against the calcium-free structure, respectively. Global LDDT scores for the experimental structures are $49 \%$ for all atoms and $56 \%$ for $C_{a}$ only. 

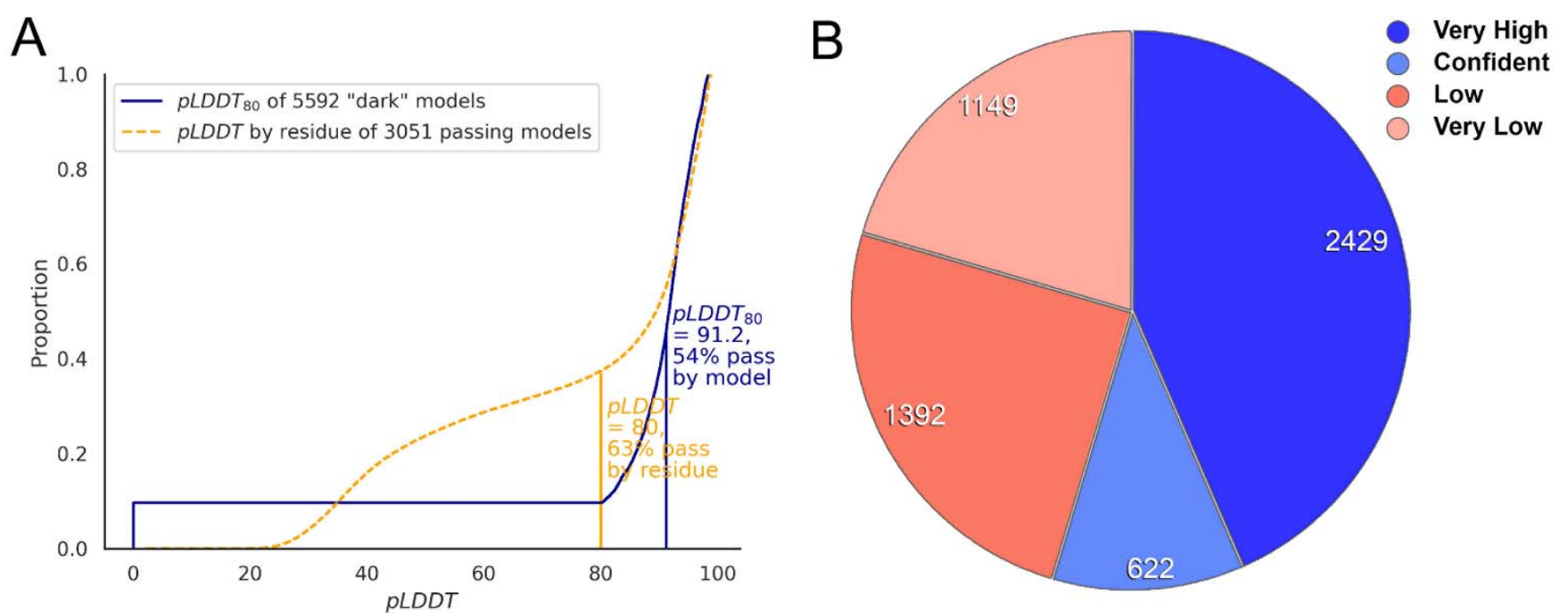

Figure 4. Fraction of the dark genome potentially illuminated by AF2 models. A) Of the set of 5592 unique "dark" proteins with AF2 models, 3051 (54\%) pass the proposed selection criteria of pLDDT80 greater to or equal to 91.2 while having at least 20 residues with $p L D D T$ $>=80$. B) Pie chart illustrating AF2 model quality according to pLDDT80-derived criteria (see Supplementary Information): 3051 (54\%) proteins associated with "very high" or "confident" AF2 models are likely to be TBVS-ready, whereas 2541 proteins are not. 


\section{REFERENCES}

1. PAVE Poll: Americans wary of AVs but say education and experience with technology can build trust. 2020, https://pavecampaign.org/pave-poll-americans-wary-of-avs-but-say-educationand-experience-with-technology-can-build-trust/

2. National Center for Biotechnology Information. 2021, https://www.ncbi.nlm.nih.gov/

3. Maglott D, Ostell J, Pruitt KD, Tatusova T: Entrez Gene: gene-centered information at NCBI. Nucleic Acids Res 2007, 35:D26-31.

4. Piovesan A, Antonaros F, Vitale L, Strippoli P, Pelleri MC, Caracausi M: Human protein-coding genes and gene feature statistics in 2019. BMC Res Notes 2019, 12:315.

5. Burley SK, Bhikadiya C, Bi C, Bittrich S, Chen L, Crichlow GV, Christie CH, Dalenberg K, Di Costanzo L, Duarte JM, et al.: RCSB Protein Data Bank: powerful new tools for exploring 3D structures of biological macromolecules for basic and applied research and education in fundamental biology, biomedicine, biotechnology, bioengineering and energy sciences. Nucleic Acids Research 2021, 49:D437-D451.

6. Avram S, Bologa CG, Holmes J, Bocci G, Wilson TB, Nguyen D-T, Curpan R, Halip L, Bora A, Yang JJ, et al.: DrugCentral 2021 supports drug discovery and repositioning. Nucleic Acids Res 2021, 49:D1160-D1169.

DrugCentral is a public portal that provides up-to-date drug information. The current release includes newly approved active pharmaceutical ingredients (current through June 2021), pharmacokinetic properties for 1000 drugs, sex-based separation of side effects processed from FAERS (FDA Adverse Event Reporting System), and a machine learning platform that estimates anti-SARS-CoV-2 activities, REDIAL-2020.

7. Oprea TI, Bologa CG, Brunak S, Campbell A, Gan GN, Gaulton A, Gomez SM, Guha R, Hersey A, Holmes J, et al.: Unexplored therapeutic opportunities in the human genome. Nature Reviews Drug Discovery 2018, 17:317-332.

8. Sheils TK, Mathias SL, Kelleher KJ, Siramshetty VB, Nguyen D-T, Bologa CG, Jensen LJ, Vidović D, Koleti A, Schürer SC, et al.: TCRD and Pharos 2021: mining the human proteome for disease biology. Nucleic Acids Res 2021, 49:D1334-D1346.

The Target Central Resource Database (TCRD) is an open-access resource developed as a part of the IDG program and jointly serves as the knowledge hub for over 20000 human protein targets (collating information from several gene/protein data sources). And Pharos is the web interface to browse the TCRD. Pharos recently added AF2 structures to each protein target, and continues to add useful information to empower users to find new areas of study in the druggable genome.

9. Ochoa D, Hercules A, Carmona M, Suveges D, Gonzalez-Uriarte A, Malangone C, Miranda A, Fumis L, Carvalho-Silva D, Spitzer M, et al.: Open Targets Platform: supporting systematic drug-target identification and prioritisation. Nucleic Acids Res 2021, 49:D1302-D1310. 
10. Carter AJ, Kraemer O, Zwick M, Mueller-Fahrnow A, Arrowsmith CH, Edwards AM: Target 2035: probing the human proteome. Drug Discov Today 2019, 24:2111-2115.

11. Jumper J, Evans R, Pritzel A, Green T, Figurnov M, Ronneberger O, Tunyasuvunakool K, Bates R, Žídek A, Potapenko A, et al.: Highly accurate protein structure prediction with AlphaFold. Nature 2021, 596:583-589.

The authors describe the successful development/methods of AF2, including the incorporation of evolutionary information through multiple-sequence alignments and the use of SE(3) transformers to handle rotational and translational symmetries in an equivariantattention fashion. Based on early citations, it appears this paper is likely to become one of the most cited papers in contemporary science. Of particular interest is Fig. $2 \mathrm{~A}$ of this paper which shows the distribution of mean atomic root-mean-square deviations between models and a subset (those that did not cluster with PDBclust) of crystal structures deposited since AF2 was trained.

12. Tunyasuvunakool K, Adler J, Wu Z, Green T, Zielinski M, Žídek A, Bridgland A, Cowie A, Meyer $\mathrm{C}$, Laydon A, et al.: Highly accurate protein structure prediction for the human proteome. Nature 2021, 596:590-596.

This paper documents the deposition of 350,000 structural models produced by AlphaFold2, including models for $99 \%$ of the human genome. Of particular interest is the discussion and how disordered regions in proteins correlate with confidence scores.

13. Jumper J, Evans R, Pritzel A, Green T, Figurnov M, Ronneberger O, Tunyasuvunakool K, Bates R, Žídek A, Potapenko A, et al.: Applying and improving AlphaFold at CASP14. Proteins 2021, doi:10.1002/prot.26257.

14. Mariani V, Biasini M, Barbato A, Schwede T: IDDT: a local superposition-free score for comparing protein structures and models using distance difference tests. Bioinformatics 2013, 29:2722-2728.

This paper introduces the local distance difference test (LDDT), a superposition-free score that combines an agreement-based model quality measure with stereochemical plausibility checks. LDDT serves as tool for evaluating protein structure predictions.

15. Peng Z, Yan J, Fan X, Mizianty MJ, Xue B, Wang K, Hu G, Uversky VN, Kurgan L: Exceptionally abundant exceptions: comprehensive characterization of intrinsic disorder in all domains of life. Cell Mol Life Sci 2015, 72:137-151.

16. Dunker AK, Obradovic Z, Romero P, Garner EC, Brown CJ: Intrinsic protein disorder in complete genomes. Genome Inform Ser Workshop Genome Inform 2000, 11:161-171.

17. Xue B, Dunker AK, Uversky VN: Orderly order in protein intrinsic disorder distribution: disorder in $\mathbf{3 5 0 0}$ proteomes from viruses and the three domains of life. J Biomol Struct Dyn 2012, 30:137-149.

18. Ward JJ, Sodhi JS, McGuffin LJ, Buxton BF, Jones DT: Prediction and functional analysis of 
native disorder in proteins from the three kingdoms of life. J Mol Biol 2004, 337:635-645.

19. Dunker AK, Keith Dunker A, David Lawson J, Brown CJ, Williams RM, Romero P, Oh JS, Oldfield CJ, Campen AM, Ratliff CM, et al.: Intrinsically disordered protein. Journal of Molecular Graphics and Modelling 2001, 19:26-59.

20. Uversky VN, Gillespie JR, Fink AL: Why are ?natively unfolded? proteins unstructured under physiologic conditions? Proteins 2000, 41:415-427.

21. Wright PE, Dyson HJ: Intrinsically unstructured proteins: re-assessing the protein structurefunction paradigm. $J$ Mol Biol 1999, 293:321-331.

22. Tanner JJ: Empirical power laws for the radii of gyration of protein oligomers. Acta Crystallogr D Struct Biol 2016, 72:1119-1129.

23. Kufareva I, Abagyan R: Methods of protein structure comparison. Methods Mol Biol 2012, 857:231-257.

24. Zhang M, Tanaka T, Ikura M: Calcium-induced conformational transition revealed by the solution structure of apo calmodulin. Nat Struct Biol 1995, 2:758-767.

25. Vojtechovský J, Chu K, Berendzen J, Sweet RM, Schlichting I: Crystal structures of myoglobinligand complexes at near-atomic resolution. Biophys $J$ 1999, 77:2153-2174.

26. Safo MK, Ahmed MH, Ghatge MS, Boyiri T: Hemoglobin-ligand binding: Understanding Hb function and allostery on atomic level. Biochimica et Biophysica Acta (BBA) - Proteins and Proteomics 2011, 1814:797-809.

27. Meng F, Uversky VN, Kurgan L: Comprehensive review of methods for prediction of intrinsic disorder and its molecular functions. Cell Mol Life Sci 2017, 74:3069-3090.

28. Uversky VN, Oldfield CJ, Dunker AK: Showing your ID: intrinsic disorder as an ID for recognition, regulation and cell signaling. J Mol Recognit 2005, 18:343-384.

29. Galea CA, Wang Y, Sivakolundu SG, Kriwacki RW: Regulation of cell division by intrinsically unstructured proteins: intrinsic flexibility, modularity, and signaling conduits. Biochemistry 2008, 47:7598-7609.

30. Vucetic S, Xie H, Iakoucheva LM, Oldfield CJ, Keith Dunker A, Obradovic Z, Uversky VN: Functional Anthology of Intrinsic Disorder. 2. Cellular Components, Domains, Technical Terms, Developmental Processes, and Coding Sequence Diversities Correlated with Long Disordered Regions. Journal of Proteome Research 2007, 6:1899-1916.

31. Fuxreiter M, Tompa P, Simon I, Uversky VN, Hansen JC, Asturias FJ: Malleable machines take shape in eukaryotic transcriptional regulation. Nat Chem Biol 2008, 4:728-737.

32. Habchi J, Tompa P, Longhi S, Uversky VN: Introducing protein intrinsic disorder. Chem Rev 2014, 114:6561-6588. 
33. Liu J, Perumal NB, Oldfield CJ, Su EW, Uversky VN, Dunker AK: Intrinsic disorder in transcription factors. Biochemistry 2006, 45:6873-6888.

34. Deiana A, Forcelloni S, Porrello A, Giansanti A: Intrinsically disordered proteins and structured proteins with intrinsically disordered regions have different functional roles in the cell. PLoS One 2019, 14:e217889.

35. Bondos SE, Dunker AK, Uversky VN: On the roles of intrinsically disordered proteins and regions in cell communication and signaling. Cell Commun Signal 2021, 19:88.

36. Uversky VN, Oldfield CJ, Dunker AK: Intrinsically disordered proteins in human diseases: introducing the D2 concept. Annu Rev Biophys 2008, 37:215-246.

37. Midic U, Oldfield CJ, Dunker AK, Obradovic Z, Uversky VN: Unfoldomics of human genetic diseases: illustrative examples of ordered and intrinsically disordered members of the human diseasome. Protein Pept Lett 2009, 16:1533-1547.

38. Kulkarni P, Uversky VN: Intrinsically Disordered Proteins in Chronic Diseases. Biomolecules 2019,9.

39. Coskuner O, Uversky VN: Intrinsically disordered proteins in various hypotheses on the pathogenesis of Alzheimer's and Parkinson's diseases. Progress in Molecular Biology and Translational Science 2019, doi:10.1016/bs.pmbts.2019.05.007.

40. Ruan H, Sun Q, Zhang W, Liu Y, Lai L: Targeting intrinsically disordered proteins at the edge of chaos. Drug Discov Today 2019, 24:217-227.

41. Santofimia-Castaño P, Rizzuti B, Xia Y, Abian O, Peng L, Velázquez-Campoy A, Neira JL, Iovanna J: Targeting intrinsically disordered proteins involved in cancer. Cellular and Molecular Life Sciences 2020, 77:1695-1707.

42. Ruff KM, Pappu RV: AlphaFold and Implications for Intrinsically Disordered Proteins. J Mol Biol 2021, doi.org/10.1016/j.jmb.2021.167208

The authors review the top predictors of intrinsically disordered regions (IDRs) while also highlighting the future expansions in protein structure prediction led by machine learning techniques, including AF2. While there exists a large overlap between low AF2 confidence scores and experimentally determined IDRs, they note that a cautionary approach must be taken before assuming a concrete understanding of protein structure.

43. Tsafou K, Tiwari PB, Forman-Kay JD, Metallo SJ, Toretsky JA: Targeting Intrinsically Disordered Transcription Factors: Changing the Paradigm. Journal of Molecular Biology 2018, 430:2321-2341.

44. Sergey O: ColabFold: Making Protein folding accessible to all via Google Colab! Github; 2021 https://colab.research.google.com/github/sokrypton/ColabFold/blob/main/AlphaFold2.ipynb

ColabFold is a repository of notebooks that make AlphaFold2 and other AI-driven structural 
modeling methods available to users using the latest developments. Of particular interest are the development of methods for running models employing homooligomers, custom multiplesequence alignments, and assemblies.

45. Khafizov K, Madrid-Aliste C, Almo SC, Fiser A: Trends in structural coverage of the protein universe and the impact of the Protein Structure Initiative. Proc Natl Acad Sci U S A 2014, 111:3733-3738.

46. Thornton JM, Laskowski RA, Borkakoti N: AlphaFold heralds a data-driven revolution in biology and medicine. Nat Med 2021, 27:1666-1669.

The authors comment on the variable quality of AF2 models ("good, bad and ugly") and distributions of per-model average pLDDT scores across four model organisms: human, Trypanosoma cruzi, Mycobacterium tuberculosis and Escherichia coli. They note that $M$. tuberculosis and E. coli have twice as many "very high" confidence scores compared to those in and $T$. cruzi, due to differences in average sequence lengths. Given our findings on the large effects that disorder has on untruncated confidence scores, these differences could also reflect higher amounts of disordered regions in eukaryotes compared with bacteria.

47. Database APS: AlphaFold Protein Structure Database. 2021, https://alphafold.ebi.ac.uk

48. Hauser M, Steinegger M, Söding J: MMseqs software suite for fast and deep clustering and searching of large protein sequence sets. Bioinformatics 2016, 32:1323-1330. 


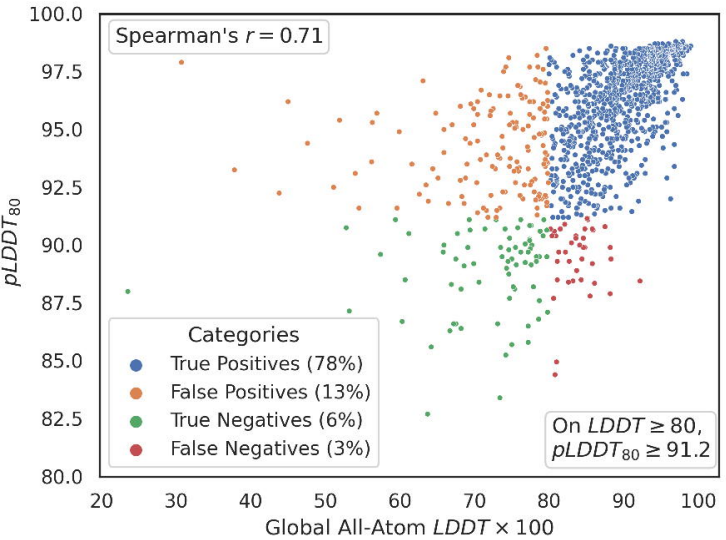

